\title{
Notoginsenoside $R 1$ attenuates glucose-induced podocyte injury via the inhibition of apoptosis and the activation of autophagy through the PI3K/Akt/mTOR signaling pathway
}

\author{
GUODONG HUANG ${ }^{1,2^{*}}$, BINGYU ZOU ${ }^{3 *}$, JIANZHEN LV $^{4 *}$, TONGYU LI ${ }^{4}$, GUOLI HUAI ${ }^{5}$, \\ SHAOWEI XIANG ${ }^{1}$, SHILONG LU ${ }^{4}$, HUAN LUO ${ }^{1}$, YAPING ZHANG ${ }^{1}$, YI JIN ${ }^{6}$ and YI WANG ${ }^{5,7}$ \\ ${ }^{1}$ Department of Nephrology, Ruikang Hospital Affiliated to Guangxi University of Chinese Medicine, Nanning, \\ Guangxi 530011; ${ }^{2}$ Center for Liver and Intestinal Surgery, Central South University, Changsha, Hunan 410008; \\ ${ }^{3}$ Department of Gynecology, Sichuan Academy of Medical Science and Sichuan Provincial People's Hospital, Chengdu, \\ Sichuan 610072; ${ }^{4}$ School of Basic Medical Science, Guangxi University of Chinese Medicine, Nanning, Guangxi 530001; \\ ${ }^{5}$ Department of Biomedical Engineering, Medical School of University of Electronic Science and Technology of China, \\ Chengdu, Sichuan 610054, P.R. China; ${ }^{6}$ Center for Perinatal Research, Research Institute at Nationwide Children's Hospital, \\ Columbus, OH 43215, USA; 7 Department of Pharmacy, Sichuan Academy of Medical Science \\ and Sichuan Provincial People's Hospital, Sichuan, Chengdu 610072, P.R. China
}

Received September 6, 2015; Accepted January 11, 2017

DOI: $10.3892 /$ ijmm.2017.2864

\begin{abstract}
Injury to terminally differentiated podocytes contributes ignificantly to proteinuria and glomerulosclerosis. The aim of this study was to examine the protective effects of notoginsenoside R1 (NR1) on the maintenance of podocyte number and foot process architecture via the inhibition of apoptosis, the induction of autophagy and the maintenance $\mathrm{pf}$ podocyte biology in target cells. The effects of NR1 on conditionally immortalized human podocytes under high glucose conditions were evaluated by determining the percentage apoptosis, the percentage autophagy and the expression levels of slit diaphragm proteins. Our results revealed that NR1 protected the podocytes against high glucose-induced injury by decreasing apoptosis, increasing autophagy and by promoting cytoskeletal recovery. The phosphoinositide 3-kinase (PI3K)/Akt/mammalian target
\end{abstract}

Correspondence to: Dr Yi Wang, Department of Pharmacy, Sichuan Academy of Medical Science and Sichuan Provincial People's Hospital, 32 First Ring Road, Chengdu, Sichuan 610072, P.R. China

E-mail:w_yi@yahoo.com

Dr Yi Jin, Center for Perinatal Research, Research Institute at Nationwide Children's Hospital, 575 Children's Crossroads, Columbus, OH 43215, USA

E-mail: jinyi67@yahoo.com

${ }^{*}$ Contributed equally

Key words: podocyte, apoptosis, autophagy, notoginsenoside R1, $\mathrm{PI} 3 \mathrm{~K} / \mathrm{Akt} / \mathrm{mTOR}$ pathway of rapamycin (mTOR) signaling pathway was further investigated in order to elucidate the mechanisms responsible for the protective effects of NR1 on podocytes. Our data indicated that treatment with NR increased the phosphorylation levels of PI3K, Akt and mTOR, leading to the activation of the PI3K/ Akt/mTOR signaling pathway in podocytes. To the best of our knowledge, this is the first in vitro study to demonstrate that NR1 protects podocytes by activating the PI3K/Akt/mTOR pathway.

\section{Introduction}

Diabetic nephropathy (DN) is the leading cause of chronic kidney disease, which ultimately progresses to end-stage renal failure. In early DN, glomerular abnormalities are observed, including the increase in glomerular filtration rate and albuminuria $(1,2)$. Accumulating evidence indicates that podocyte injury contributes to these pathological changes $(3,4)$. Clinical data also demonstrate that podocyte integrity is impaired in individuals with type 1 and 2 diabetes $(5,6)$. It is widely acknowledged that the podocyte slit diaphragm plays a critical role in establishing the selective permeability of the glomerular filtration barrier (7). In our previous study (8), we demonstrated the in vivo renal protective role of notoginsenoside R1 (NR1) through the regulation of phosphoinositide 3-kinase (PI3K)/ Akt signaling. However, the mechanisms through which NR1 regulates this signaling pathway remain unknown. Therefore in this study, we performed in vitro experiments and focused on podocyte slit diaphragm proteins that are important in maintaining podocyte integrity. Podocyte decrement is also the leading cause of these pathological changes. It has been reported that podocytes are highly specialized, terminally differentiated cells with limited replicative capacity (9). In both type 1 and 2 diabetes, the decreased podocyte number is a major pathophys- 
iological precursor to the development of proteinuria $(10,11)$. Taken together, these data indicate that the ability to maintain podocyte integrity and podocyte number seems to be of pivotal importance in protecting against diabetes.

It is acknowledged that the search for more effective therapeutic drugs to prevent podocyte injury and to maintain podocyte number is a great challenge. As herbal medicine has been clinically used in the treatment of DN for centuries in China, in this study, we investigated the role of NR1 (extracted from Panax notoginseng) on podocytes and the underlying mechanisms. It has been demonstrated that NR1 attenuates renal ischemia-reperfusion injury in rats (12). It has also been demonstrated that NR1 attenuates the glucose-induced impairement of podocyte adhesive capacity and subsequent podocyte depopulation in rats through the upregulation of $\alpha 3 \beta 1$ integrin (13). However, it is not clear whether NR1 has any effect on the number of podocytes by affecting apoptosis and inducing autophagy and podocyte integrity in addition to its effect on podocyte adhesion (13).

Although a large body of evidence has indicated that apoptosis contributes greatly to the podocyte depopulation, autophagy has also been shown to be important in preservating the number of podocytes (14-17). It has also been demonstrated that in renal injury, autophagy plays a protective role by delaying the progression of podocytopathies (18). In our previous in vivo study (8), using mice, we reported that NR1 protects podocytes from injury via the PI3K/Akt pathway. However, which site activation/ phosphorylation of the PI3K/Akt pathway is regulated by NR1 remains unkown. Among several signaling pathways that are functional in podocytes, it has been reported that decreased the phosphorylation of Akt is associated with podocyte loss in early DN (19). It has been demonstrated that in rats with puromycin aminonucleoside (PAN) nephropathy, the activation of the PI3K/Akt signaling inhibits podocyte apoptosis (20). Furthermore, it has been reported that $\mathrm{Na}^{+} / \mathrm{H}+$ exchanger-1 (NHE-1) attenuates podocyte injury via the PI3K/Akt pathway by inducing the activation of autophagy (21). It is thus possible that the PI3K/Akt pathway may be involved in both apoptosis and autophagy during the process of podocyte injury. Moreover, previous studies have demonstrated that the inhibition of mammalian target of rapamycin (mTOR) protects podocytes from injury and prevents DN through the regulation of autophagy $(22,23)$. Due to the importance of the PI3K/Akt/mTOR pathway in apoptosis and autophagy, in the present study, we investigated the protective effects of NR1 on podocytes, as well as whether and how the PI3K/Akt/mTOR signaling pathway is regulated by NR1. Moreover, we wished to determine whether the PI3K/Akt/mTOR signaling pathway is involved in the protective effects of NR1 on podocytes, and to elucidate the mechanisms through which Akt regulates both apoptosis and autophagy in NR1-treated podocytes.

\section{Materials and methods}

Chemicals. NR1 was purchased from Sigma Chemical Co. (St. Louis, MO, USA), and the purity of NR1 was $>98 \%$. The autophagy inhibitor, 3-methyladenine (3-MA), monodansylcadaverine (MDC), and 2',7'-dichlorofluorescein diacetate (DCF) were also purchased from Sigma Chemical Co. Small interfering RNAs (siRNAs) against human Beclin 1 and scramble siRNA were purchased from Cell Signaling Technology, Inc. (Beverly, MA, USA). Hoechst 33342, the Click-iT TUNEL Alexa Fluor 488 Imaging Assay kit for microscopy and high-content screening (HCS), SYBR-Green PCR Master Mix, NuPage Novex 10\% Bis-Tris gel, NuPage transfer buffer, TRIzol, the High Capacity cDNA Reverse Transcription kit, the Dead Cell Apoptosis kit with Annexin V Alexa Fluor 488 and propidium iodide (PI) and 4',6-diamidino2-phenylindole (DAPI) were purchased from Invitrogen Life Technologies (Carlsbad, CA, USA). Polyvinylidene fluoride membranes were purchased from Pall Life Sciences (Port Washington, NY, USA). Immobilon Western Chemiluminescent HRP Substrate was purchased from Millipore Corp. (Billerica, MA, USA). The CellTiter-Glo luminescent cell viability assay kit and terminal deoxynucleotidyltransferase-mediated dUTP nick-end labeling (TUNEL) assay kit were purchased from Promega Corp. (Madison, WI, USA)

The following antibodies were purchased from Santa Cruz Biotechnology, Inc. (Dallas, TX, USA): nephrin (sc-377246), desmin (sc-65983), pro-caspase-3 (sc-7148), pro-caspase-9 (sc-56073), cleaved caspase-9 (sc-22182), Bax (sc-493), Bid (sc-135847), Bcl-2 (sc-492), Bcl-xL (sc-8392), poly(ADP-ribose) polymerase (PARP; sc-7150) and $\beta$-actin (sc-47778). Besides, rat anti-mouse podocalyxin (PCX) antibody (MAB1556) was purchased from R\&D Systems (Minneapolis, MN, USA). In addition, antibodies against podocin (ab50339), CD2AP (ab84829), active caspase-3 (ab49822), Beclin (ab62557), PI3K p85 (ab189403), phosphorylated (p-)Beclin 1 (S234; ab183335), mTOR (ab2732), p-mTOR (S2448; ab109268), p-mTOR (S2481; ab137133), p-PI3K p85 (Y607; ab182651) were from Abcam (Cambridge, UK). Antibodies to light chain 3 (LC3; \#12741), Akt (pan; \#2920), cleaved caspase-9 (\#9509), p-Akt (T308; \#9275), p-Akt (S473, \#4060), p-p70S6K (Thr389; \#9206) and p-eukaryotic translation initiation factor 4E-binding protein 1 (4E-BP1; T37/46; \#2855) were all purchased from Cell Signaling Technology, Inc. Goat anti-rabbit IgG antibody conjugated with HRP (\#11-035-003), goat anti-mouse IgG antibody conjugated with HRP (\#115-035-003), and goat anti-rabbit IgG antibody conjugated with Cy3 (\#111165-144) were purchased from Jackson ImmunoResearch Laboratories, West Grove, PA, USA.

Cell culture and treatment with NR1. Conditionally immortalized human podocytes were kindly provided by Dr Guisen Li (Division of Nephrology, from the Sichuan Provincial People's Hospital) and were cultured as previously described (24). In brief, in order to maintain an undifferentiated proliferative state, podocytes were cultured at $33^{\circ} \mathrm{C}$ with RPMI-1640 medium (Life Technologies) supplemented with $10 \%$ fetal bovine serum (Life Technologies). Before use, when the cells had reached $70-80 \%$ confluence, they were cultured at $37^{\circ} \mathrm{C}$ to reach differentiation. The podocytes were divided into the following groups: i) the low glucose (LG) group: the cells were incubated in RPMI-1640 containing $5 \mathrm{mM}$ D-glucose; ii) the high glucose (HG) group: the cells were incubated in RPMI-1640 containing $30 \mathrm{mM}$ D-glucose; iii) the NR1-treated (NR1) group: the cells were incubated in 
RPMI-1640 containing $30 \mathrm{mM}$ D-glucose and then treated with $20 \mu \mathrm{M}$ NR1 for $24 \mathrm{~h}$.

Cell viability and cell morphology. To ensure that NR1 can abolish the growth inhibitory effects of high glucose on podocytes, podocyte viability was measured within $24 \mathrm{~h}$ of exposure to a series of NR1 concentrations $(0,1,5,10,20,50$ and $100 \mu \mathrm{M})$. The number of viable cells was assessed by CellTiter-Glo luminescent cell viability assay kit according to the manufacturer's instructions in triplicate. Cell morphology was also observed under a light microscope (Leica Microsystems GmbH, Wetzlar, Germany).

Hoechst staining. The podocytes were grown to $80 \%$ confluence on 6-well plates under growth-restricted conditions and treated with low glucose, high glucose, or a combination of both high glucose and $20 \mu \mathrm{M}$ NR1 for $24 \mathrm{~h}$. Hoechst 33342 was added to achieve a final concentration of $10 \mu \mathrm{M}$ and the cells were analyzed after 5 min of Hoechst staining. Apoptotic cells with condensed chromatin were manually counted for a minimum of 20 high-powered fields per experimental condition by two blinded technicians. The number of apoptotic nuclei was expressed as a percentage of the total number of apoptotic nuclei.

Annexin V-PI assay. Following the experimental treatments, the differentiated human podocytes were dispersed from 6-well culture plates, collected in PBS, washed with FACS buffer, and stained using the Dead Cell Apoptosis kit with Annexin V-PI following the manufacturer's instructions. Cells $\left(10^{4}\right)$ were acquired using a FACScan flow cytometer (Becton-Dickinson, San Jose, CA, USA). The number of apoptotic cells was analyzed using CellQuest software 3.3 (Becton-Dickinson) and was expressed as a percentage of the number of total cells.

TUNEL assay. Podocyte apoptosis was analyzed by TUNEL assay according to the manufacturer's recommendations. Following fixation in $4 \%$ buffered paraformaldehyde (PFA), the cells were incubated with terminal deoxynucleotidyl transferase using FITC-labeled nucleotides. DAPI served as a nuclear counterstain (blue). TUNEL-positive cells (green) in 15-20 randomly selected fields were manually counted out of the total number of cells.

Caspase assay. Podocyte apoptosis was also assessed by measuring the activity of caspase-3 using the caspase- 3 activity kit (Beyotime Institute of Biotechnology, Haimen, China) according to the manufacturer's instructions. In brief, the cells were lysed, and the supernatant was collected, quantified and incubated with the caspase-3-specific color substrate Ac-DEVD-pNA. Caspase-3 activity was determination by measuring optical density at OD400 $\mathrm{nm}$.

Immunofluorescence staining. The podocytes were grown in triplicate on type I collagen-coated glass coverslips, fixed with 4\% PFA, and permeabilized with Triton X-100. Following the removal of the remaining Triton X-100, the cells were incubated with anti-active caspase- 3 antibodies overnight, followed by Cy3-conjugated secondary antibody. For nuclear staining, the cells were stained with DAPI. For the negative contro, PBS was used instead of the primary antibody. The total cell population and the number of cleaved caspase-3-positive cells were manually counted.

MDC assay and Beclin knockdown. Following incubation with $20 \mu \mathrm{M}$ NR1 for the indicated periods of time, the podocytes were cultured with $0.05 \mathrm{mM} \mathrm{MDC}$ at $37^{\circ} \mathrm{C}$ for $60 \mathrm{~min}$. The fluorescence intensity of the cells was analyzed by flow cytometry. The cells were respectively transfected with Beclin 1 siRNA and scramble siRNA at $33 \mathrm{nM}$ using DharmaFect (\#T-2004; Dharmacon, Lafayette, CO, USA) according to the manufacturer's instructions (25). The transfected cells were used for subsequent experiments $24 \mathrm{~h}$ later.

Western blot analysis. The cells were lysed and the protein lysates were centrifuged at $12,000 \mathrm{rpm}$ for $30 \mathrm{~min}$. Supernatants were collected and loaded on a SDS-PAGE gel (Bio-Rad Laboratories, Inc., Hercules, CA, USA) for electrophoresis. Following electrophoresis, the proteins were transferred onto PVDF membranes in transfer buffer. The membranes were blocked with 5\% non-fat dry milk in TBS for $1 \mathrm{~h}$. The blots were incubated overnight at $4^{\circ} \mathrm{C}$ with primary antibodies at the dilutions recommended by the manufacturer, followed by incubating with horseradish peroxidase (HRP)-conjugated secondary antibody, and then visualized by Immobilon Western Chemiluminescent HRP substrate (26).

Reverse transcription-quantitative PCR (RT-qPCR). RNA was extracted using the RNeasy micro kit (Qiagen, Hilden, Germany) and reverse transcribed [1 $\mu \mathrm{g}$ total RNA, $1 \mu 1$ random primer (50 $\mu \mathrm{mol} / 1$; Applied Biosystems, Foster City, CA, USA), $1 \mathrm{X}$ reverse transcriptase buffer, and 10 units reverse transcriptase (Promega Corp.) in a total volume of $20 \mu \mathrm{l}]$. The RNA and primer were heated to $72^{\circ} \mathrm{C}$ and slowly cooled before reverse transcription at $42^{\circ} \mathrm{C}$ for $1 \mathrm{~h}$. The room temperature reaction was then diluted to $100 \mu \mathrm{l}$ with RNase-free water (Applied Biosystems) as previously described (27). For quantitative PCR (qPCR), 2.5\% of the total room temperature reaction was used as input for PCR using SYBR-Green Master Mix (Applied Biosystems) and templates were subjected to 36 rounds of PCR $\left(94^{\circ} \mathrm{C}\right.$ for $30 \mathrm{sec} ; 56^{\circ} \mathrm{C}$ for $30 \mathrm{sec} ; 72^{\circ} \mathrm{C}$ for $1 \mathrm{~min}$ ) on an ABI 7900 Real-Time PCR system. As previously described (28-30), the following primers were used: podocin forward, 5'-GGCTGTGGAGGCTGAAGC-3' and reverse 5'-CTCAGAAGCAGCCTTTTCCG-3'; nephrin forward, 5'-CGGTACAGGATCTGGCTGTT-3' and reverse 5'-CTCTC TCCACCTCGTCATACA-3'; podocalyxin forward, 5'-CTAC GGACTCATCTAACAAA-3' and reverse, 5'-AGATAACC GATGACGGTA-3'; desmin forward, 5'-CAGAATTGAA TCTCTCAACG-3' and reverse, 5'-CTTCAGAAATGTT CTTAGCC-3'; CD2AP forward, 5'-AACAGATACCGAA GGTAAAA-3' and reverse, 5'-ATCAAATGGATTATCT CCCC-3' and GAPDH forward, 5'-TGGTCACCAGGGCT GCTT-3' and reverse, 5'-AGCTTCCCGTTCTCAGCCTT-3'. The $2^{-\Delta \Delta \mathrm{Ct}}$ method was used to calculate the relative amount of the target RNAs, as previously described (31).

Statistical analysis. Data are expressed as the means \pm SEM from at least 3 independent experiments. Statistical significance was determined by one-way ANOVA with Bonferroni correc- 
A

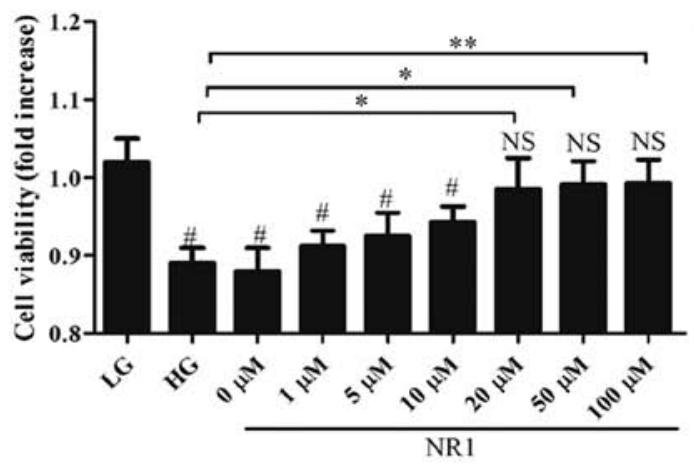

C

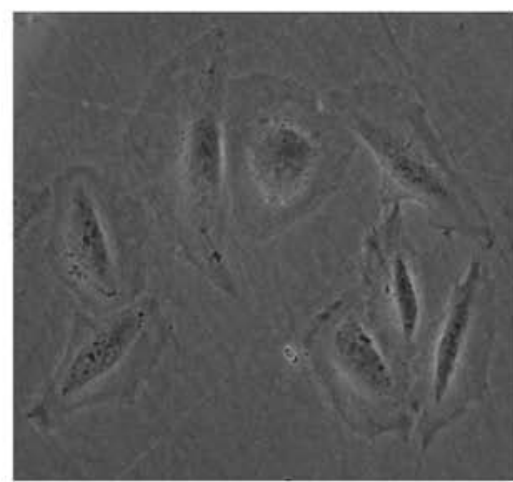

B

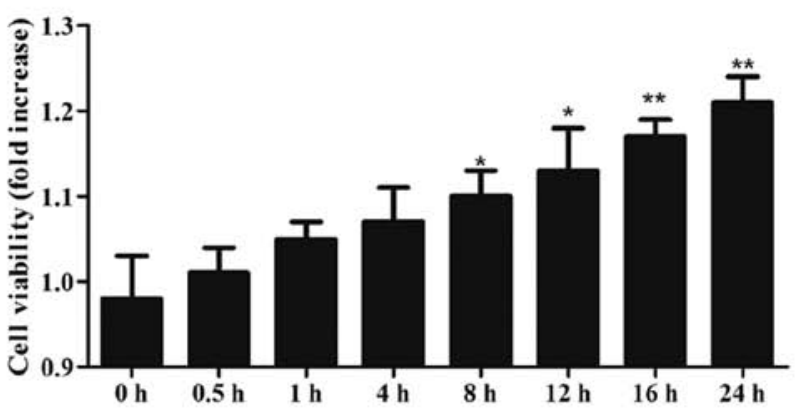

HG

NR1
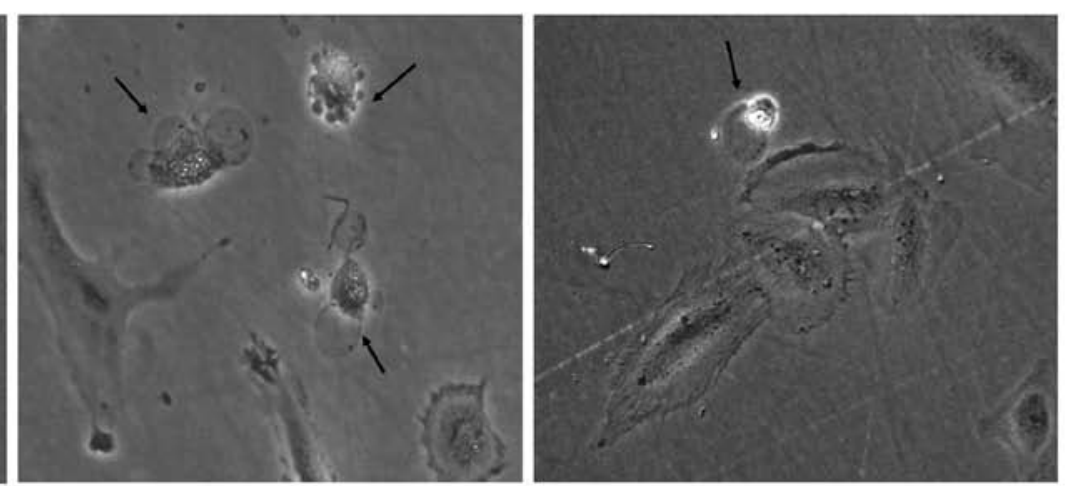

Figure 1. Notoginsenoside R1 (NR1) increases the viability of podocytes cultured under high glucose conditions. (A) Dose-dependent effect of NR1 on the viability of podocytes. (B) Time-course analysis of the effects of NR1 on cell viability. (C) Cell morphological changes. All images are at $\mathrm{x} 400$ magnification, and the arrows indicate membrane blebbing. Data are shown as the means \pm SEM from at least 3 independent experiments, ${ }^{*} \mathrm{p}<0.05,{ }^{* *} \mathrm{p}<0.01,{ }^{\#} \mathrm{p}<0.05 \mathrm{vs}$. LG group; NS, no significant difference vs. LG group.

tion for thye comparison of 3 groups or the Student's t-test for the comparison of 2 groups.

\section{Results}

NR1 protects against high glucose-induced podocyte apoptosis. Accumulating evidence has indicated that podocyte apoptosis is an early step in the progression of DN (15-17). We thus hypothesized that NR1 may exert protective effects on podocytes by inhibiting apoptosis. Cell viability was measured to determine the dose response of podocytes to NR1 (Fig. 1A). $\mathrm{NR} 1$ at the concentration of $20 \mu \mathrm{M}$ was proven to be effective in protecting the podocytes, as evidenced by a significant increase in cell viability compared to the group exposed to high glucose. Subsequent time-course experiments using $20 \mu \mathrm{M}$ NR1 revealed that NR1 treatment increased cell viability significantly after $8 \mathrm{~h}$ and resulted in a $22.7 \%$ increase at $24 \mathrm{~h}$ (Fig. 1B). NR1 restored cell viability in the HG-treated podocytes, indicating its protective effects on podocytes. To induce significant changes in cell viability, NRI treatment at $20 \mu \mathrm{M}$ for $24 \mathrm{~h}$ of the HG-treated podocytes was used in all subsequent experiments.

To determine whether NR1 protects podocytes from apoptosis, cell morphology was assessed under a light miscroscope (Fig. 1C). The podocytes in the HG group exhibited marked apoptotic morphological changes, including membrane blebbing, cell volume reduction and rounding. These morphological changes were attenuated in the podocytes in the NR1 group (Fig. 1C). Moreover, the percentage apoptosis was measured by Hoechst 33342 staining (Fig. 2A and B). As shown in Fig. 2A, considerably greater DNA fragmentation in the nuclei was observed in the podocytes in the HG group than in those in the LG group, and normal, round and homogeneously stained nuclei were observed in the NR1-treated podocytes. Statistical analysis also indicated that exposure to high glucose resulted in increased apoptosis events that were attenuated by NR1 (Fig. 2B). There was no statistically significant difference in the percentage apoptosis between the cells in the LG group and those in the NR1 group. In order to validate the results obtained by Hoechst 33342 staining, Annexin V-PI staining and FACS analysis were performed as a second independent method to measure apoptosis (Fig. 3). The FACS data revealed similar results as the Hoechst staining. In addition, TUNEL staining was performed as a third independent method to measure apoptosis (Fig. 4A), and the numbers of TUNELpositive cell percentages were manually calculated (Fig. 4B). Consistent with the other two methods, the TUNEL staining results also demonstrated that high glucose induced the apoptosis of podocytes and that this effect was reversed by NR1. Taken together, these data convincingly demonstrated that NR1 inhibits podocyte apoptosis in vitro.

Effects of NRI on foot process effacement. It has been previously demonstrated that the slit diaphragm bridges adjacent foot processes and functions as the ultimate molecular size filter (32). Given the clinical significance of the slit diaphragm proteins, we further analyzed the expression levels of podocin, nephrin and CD2AP. As shown in Fig. 5A, the expression levels of these proteins were significantly decreased under high glucose 
$\mathbf{A}$

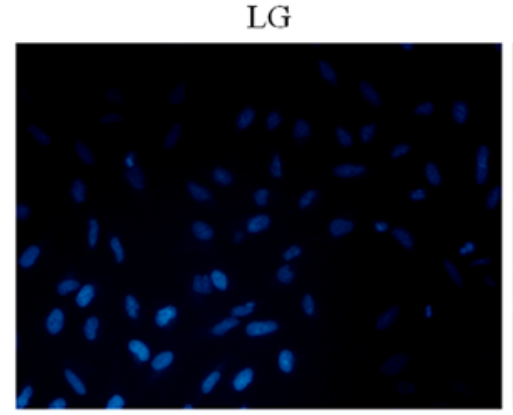

HG

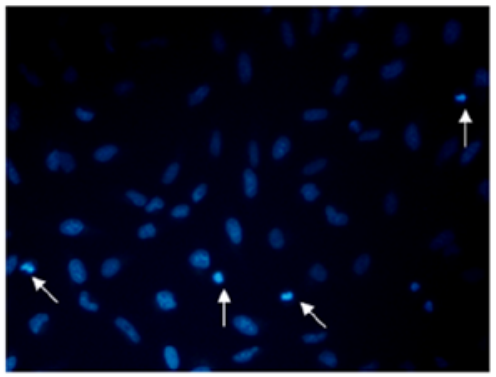

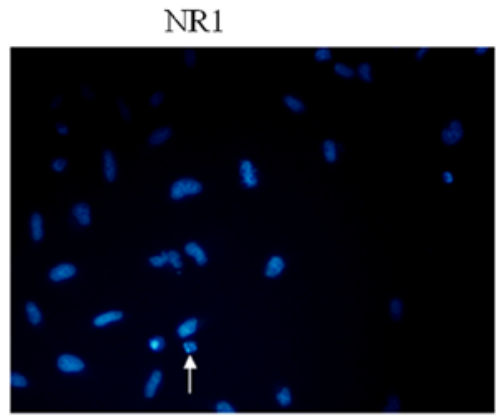

B

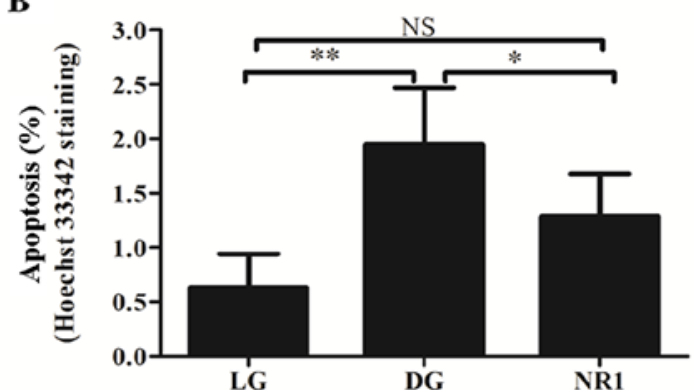

Figure 2. Assessed by Hoechst 33342 staining, notoginsenoside R1 (NR1) alleviates high glucose-induced podocyte apoptosis. (A) Hoechst 33342 staining of podocytes, and (B) apoptosis percentages quantified by Hoechst 33342 staining. All images are at x100 magnification, and the arrows indicate the apoptotic nuclei. Data are shown as the means \pm SEM from at least 3 independent experiments, ${ }^{*} p<0.05 ;{ }^{* *} \mathrm{p}<0.01 ; \mathrm{NS}$, no significant difference.
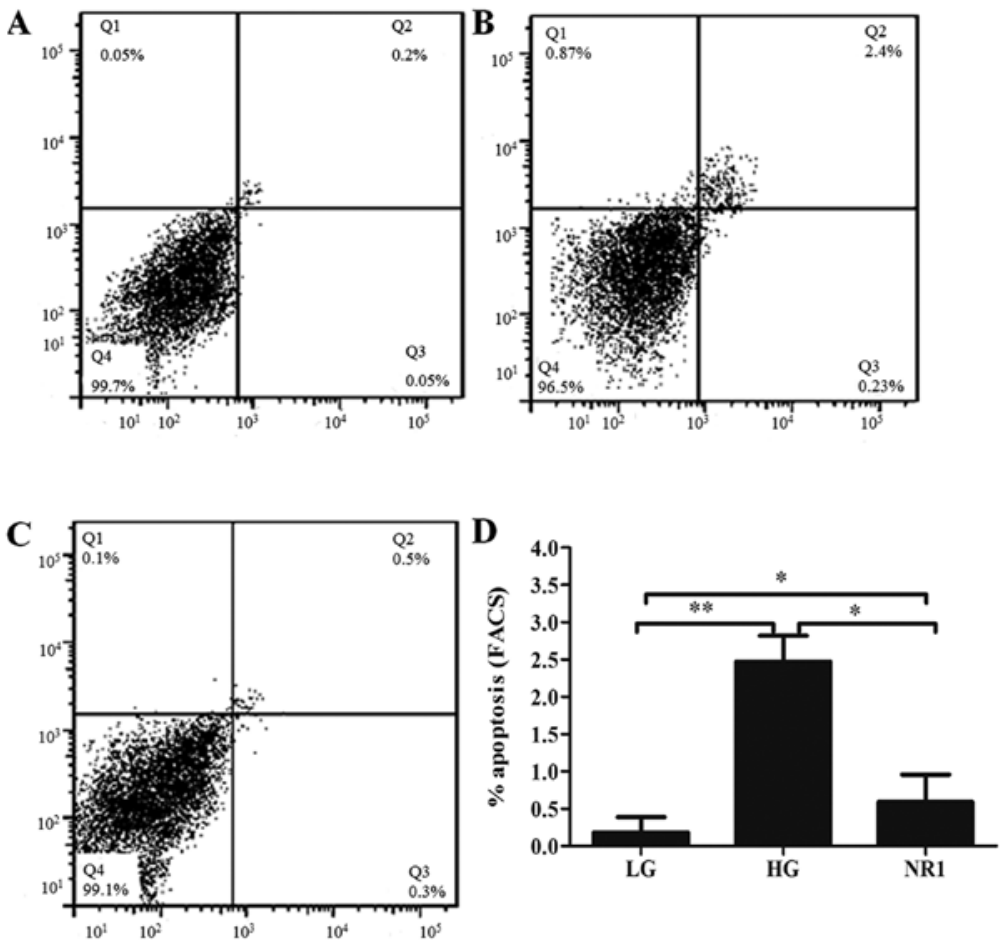

Figure 3. Annexin V-propidium iodide (PI) staining results demonstrating that notoginsenoside R1 (NR1) protects podocytes from apoptosis. Podocytes treated with (A) low glucose, (B) high glucose and (C) NR1 were fixed and then stained with Annexin V-PI. (D) Statistical analysis by flow cytometry. ${ }^{*} \mathrm{p}<0.05 ;{ }^{* *} \mathrm{p}<0.01$.

conditions and NR1 reversed this effect. Consistent with the results of western blot analysis, NR1 also restored the mRNA levels of podocin, nephrin and CD2AP in the HG-treated podocytes (Fig. 5B). As PCX inhibits cell-matrix interactions (33), and desmin is a marker of podocyte injury $(34,35)$, the protein expression of PCX and desmin was assessed by western blot analysis. Exposure to high glucose enhanced the expression levels of PCX and desmin in podocytes, while NR1 inhibited these effects (Fig. 5).

NR1 inhibits apoptosis via the caspase-dependent pathway. To further determine how NR1 inhibits podocyte apoptosis, 
A

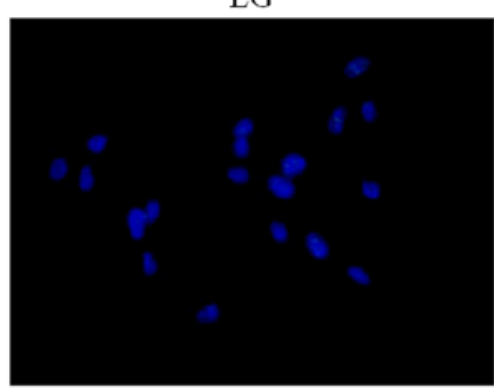

HG

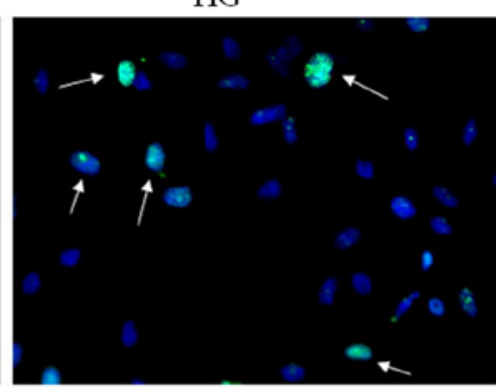

NR1

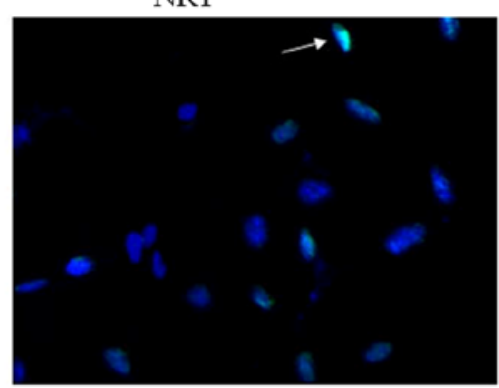

B

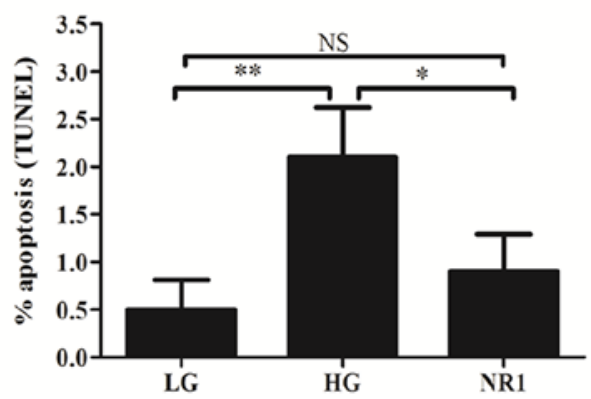

Figure 4. (A) TUNEL staining of podocytes and (B) statistical analysis demonstrating that notoginsenoside R1 (NR1) protects podocytes from apoptosis. Arrows indicate the apoptotic cells. All images are at $x 100$ magnification. Data are shown as the means \pm SEM from at least 3 independent experiments, ${ }^{*}<<0.05$; ${ }^{* *} \mathrm{p}<0.01 ; \mathrm{NS}$, no significant difference.

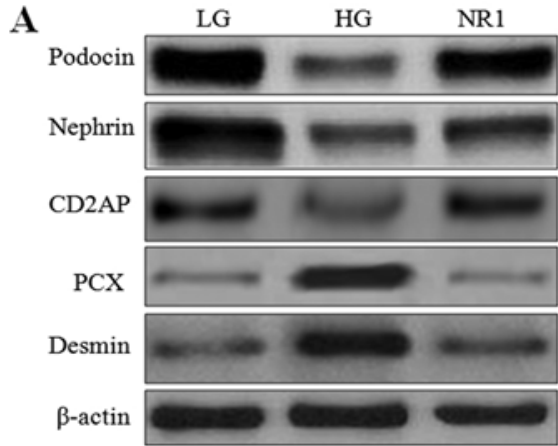

B

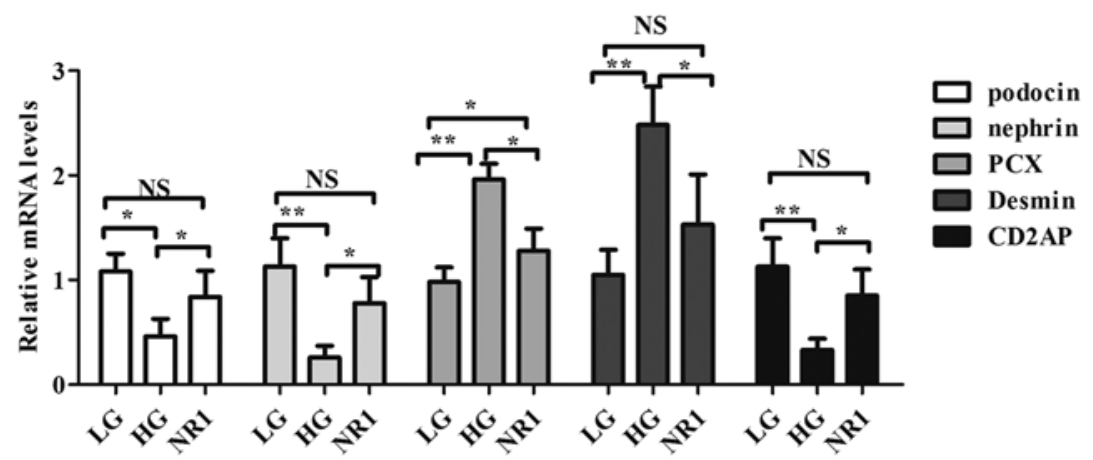

Figure 5. Notoginsenoside R1 (NR1) protects podocytes by restoring the expression of slit diaghram proteins. (A) Western blot analysis of podocyte slit diaghram proteins. Protein bands shown are representative of more than 3 independent experiments with similar results. (B) mRNA expression levels of podocin, nephrin, CD2AP, podocalyxin (PCX), and desmin quantified by RT-qPCR. Data are shown as the means \pm SEM from 3 independent experiments. ${ }^{*}$ p $<0.05$; ${ }^{* *}$ p $<0.01$; NS, no significant difference.

podocytes in the LG, HG and NR1 groups were stained with cleaved caspase- 3 antibody. The results revealed that high glucose contributed to podocyte apoptosis by increasing cleaved caspase-3 expression, while NR1 significantly inhibited the expression of cleaved caspase-3 (Fig.6A).Furthermore,caspase-3 activity assay (Fig. 6B) revealed that high glucose enhanced 
A

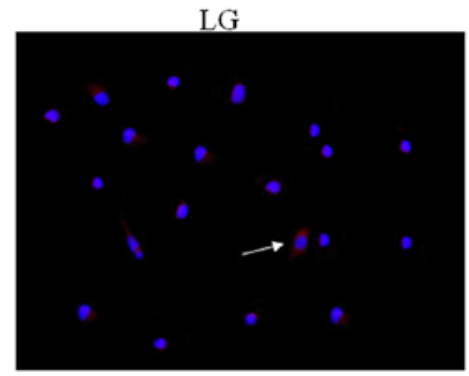

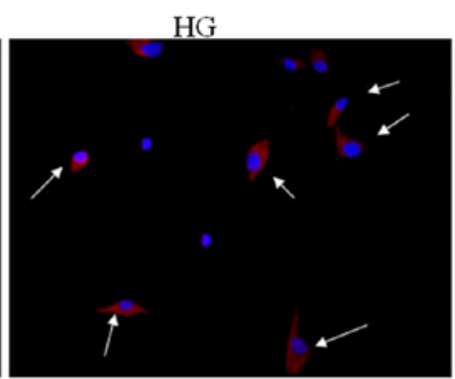

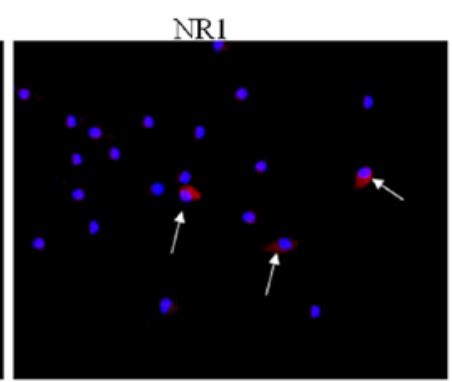

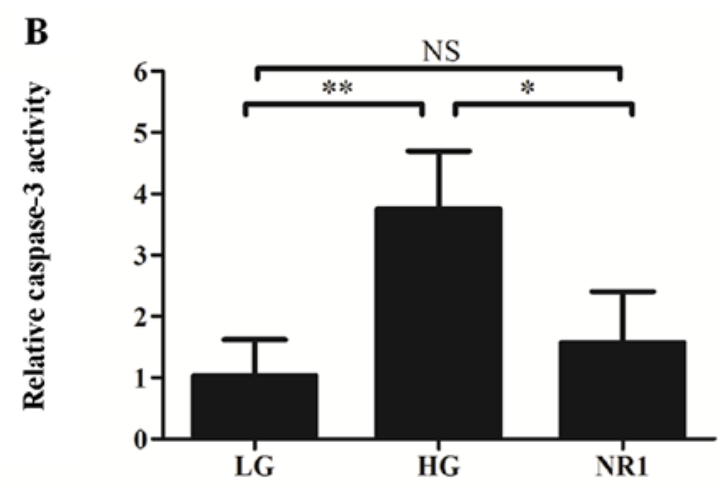

Figure 6. Notoginsenoside R1 (NR1) inhibits caspase-3 activity. (A) Immunofluorescence staining of cleaved caspase-3. White arrows indicate the cells stained positive for cleaved caspase-3. (B) Caspase-3 activity. Data are shown as the means \pm SEM from 3 independent experiments. ${ }^{*}$ p $<0.05$; ${ }^{* *}$ p $<0.01$; NS, no significant difference.

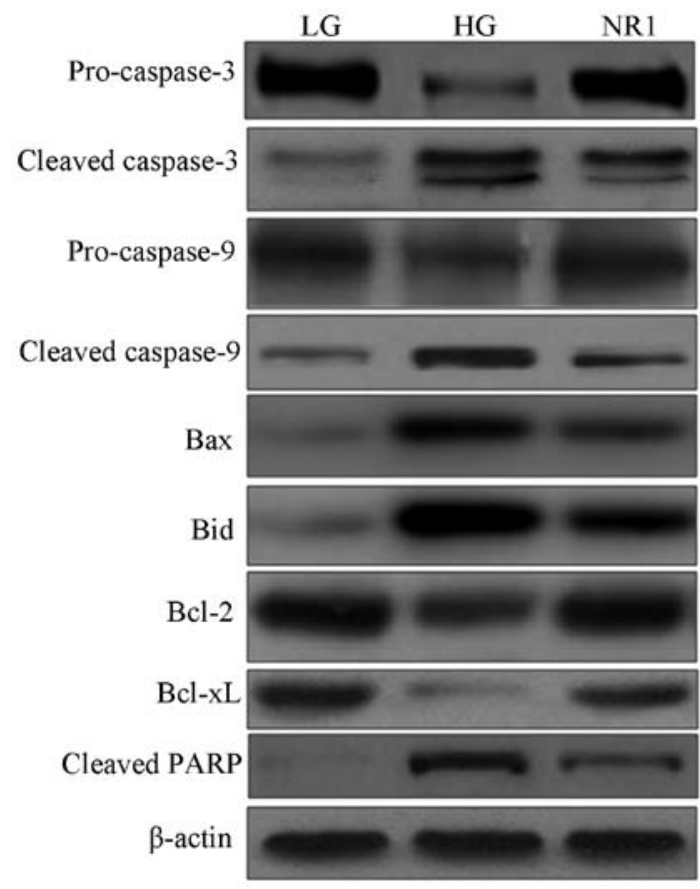

Figure 7. Western blot analysis of proteins in the caspase pathway indicating that notoginsenoside R1 (NR1) inhibits podocyte apoptosis in a caspasedependent manner. Protein bands shown are representative of more than 3 independent experiments with similar results.

caspase-3 activity in the podocytes, while NR1 significantly attenuated this effect. Western blot analysis of proteins involved in the apoptotic pathway (Fig. 7) revealed decreased expression levels of pro-caspase- 3 and -9 , and increased expression levels of cleaved caspase- 3 and -9 in the HG group podocytes, indi- cating the activation of caspase- 9 and caspase- 3 . However the cleavage of caspase- 3 and -9 was partially inhibited by NR1. In addition, the upregulation of the levels of pro-apoptotic Bax and Bid proteins, as well as the downregulation of the anti-apoptotic $\mathrm{Bcl}-2$ and $\mathrm{Bcl}-\mathrm{xL}$ proteins were observed in the podocytes in the HG group; however, NR1 downregulated the expression levels of Bax and Bid, and upregulated the expression levels of Bcl-2 and Bcl-xL in the HG-treated podocytes. Further experiments of the PARP cleavage product indicated that the exposure of the podocytes to HG increased the cleavage of PARP, and treatment with NR1 resulted in a substantial decrease in the cleavage of PARP. Taken together, our data demonstrate that NR1 significantly attenuates HG-induced podocyte apoptosis via the caspase-dependent pathway.

NR1 induces autophagy in podocytes. Increasing evidence indicates that podocytes have a higher level of constitutive autophagy than other intrinsic renal cells do, and we thus investigated the expression levels of autophagy-related proteins. Western blot ananlysis (Fig. 8A) revealed that $\mathrm{HG}$ increased the expression of LC3-II, and decreased the expression of LC3-I and Beclin 1 in podocytes. However, NR1 significantly reversed these effects on these proteins in the HG-treated cells to levels comparable to those of the LG group. To further investigate the role of autophagy in NR1 treatment, Beclin 1 was knocked down by siRNA (Fig. 8B). By measuring the MDC fluorescence intensity in the presence of Beclin 1 siRNA, we found that the silencing of Beclin 1 resulted in reduced autophagy in the NR1-treated podocytes (Fig. 8C). To further evaluate whether NR1 induces autophagy, the chemical autphagy inhibitor, 3-MA, was added to the medium of podocytes in each group. The MDC fluorescence intensity of the podocytes treated 
A

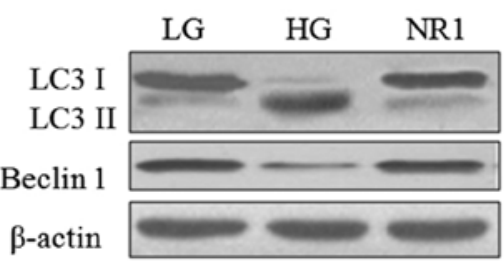

B

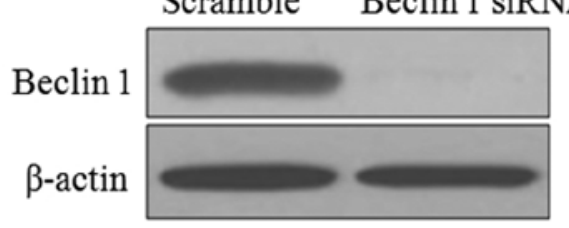

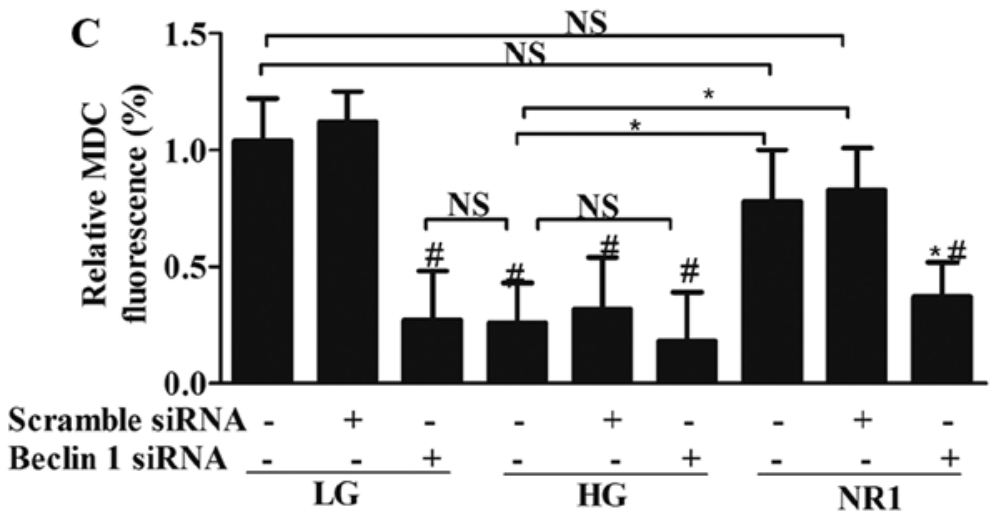

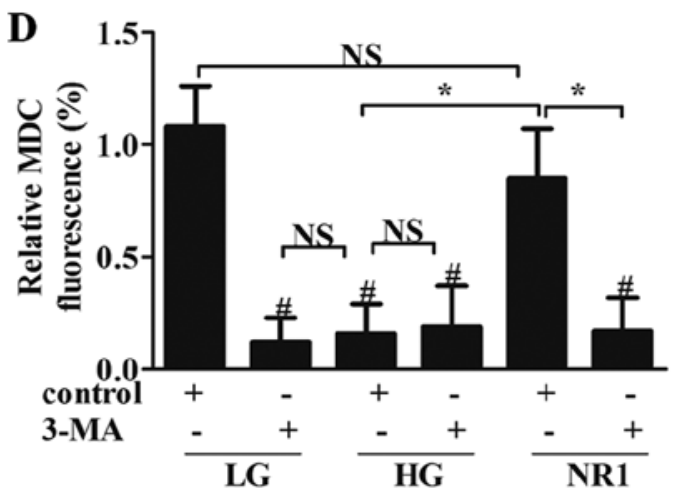

Figure 8. Notoginsenoside R1 (NR1) induces autophagy. (A) Western blot analysis of autophagy-related proteins. (B) Western blot analysis of the efficiency of Beclin 1 siRNA knockdown. The autophagy percentages were determined with either (C) Beclin siRNA or (D) with 3-MA. "p $<0.05$ vs. LG group; ${ }^{*}<<0.05$; NS, no significant difference.

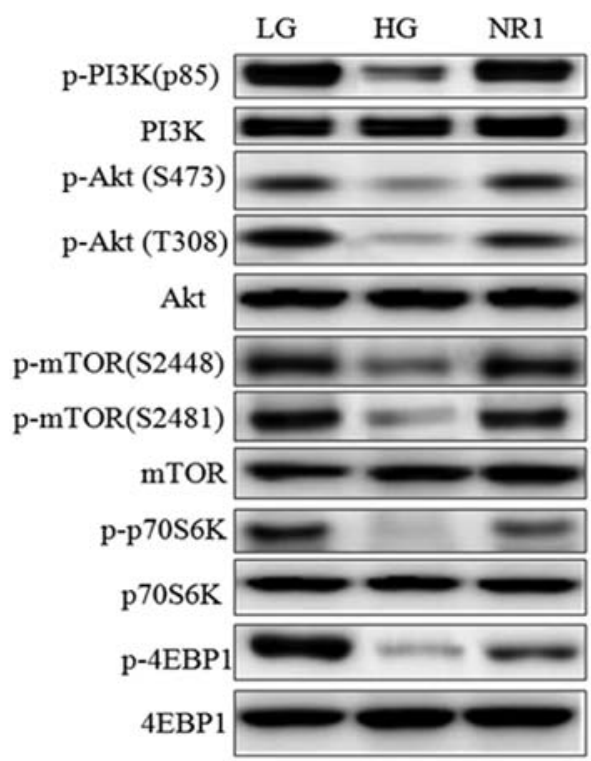

Figure 9. Western blot analysis of proteins in the PI3K/Akt/MTOR pathway indicating that notoginsenoside R1 (NR1) inhibits apoptosis and increases autography via the PI3K/Akt/MTOR pathway. Protein bands shown are representative of more than 3 independent experiments with similar results.

with 3-MA also revealed that 3-MA inhibited NR1-induced autophagy, while 3-MA had no effect on the HG-treated podocytes (Fig. 8D). These observations suggested that NR1 increased the basal autophagy that was inhibited by HG.

NR1 protects podocytes from injury via the PI3K/Akt/mTOR pathway. In order to further explore the mechanisms through which NR1 inhibits apoptosis and increases autophagy, we investigated whether the PI3K/Akt/mTOR signaling pathway is involved in these mechanisms. As shown in Fig. 9, the phosphorylation levels of PI3K (p85), Akt, mTOR and p70S6K and 4E-BP1, which are downstream genes of mTOR, were markedly decreased in the podocytes in the HG group. However, NR1 increased the phosphorylation levels of these proteins, indicating that the activation of $\mathrm{PI} 3 \mathrm{~K} / \mathrm{Akt} / \mathrm{mTOR}$ pathway may be involved in NR1 induced podocyte protection. These data suggested that the NR1-induced protective effects on podocytes were dependent on the phosphorylation and activation of the PI3K/Akt/mTOR pathway.

\section{Discussion}

In the present study, we demonstrated that NR1 preserved the podocyte number by inhibiting apoptosis and inducing autography. NR1 increased cell viability in a dose- and timedependent manner (Fig. 1A and B). NR1 significantly attenuated high glucose induced podocyte apoptosis (Fig. 1), mainly through the caspase-dependent pathway (Figs. 6 and 7). NR1 also protected podocytes from high glucose-induced damage by restoring the expression of proteins involved in podocyte slit diaphragm (Fig. 5), which was dependent on the activation of autophagy by NR1 (Fig. 8). Additionally, we found that NR1 activated the PI3K/Akt/mTOR signaling pathway, which in turn inhibited apoptosis and enhanced autography (Fig. 9). Taken together, our studies suggest that NR1 protects podocytes from high glucose-induced injury by inhibiting apoptosis and promoting autophagy via the PI3K/Akt/mTOR pathway.

Podocytes, terminally differentiated cells, have a very limited capacity for division and replacement. Therefore, podocyte damage and decreased podocyte number contribute to proteinuria and to the development of glomerulosclerosis. 
Emerging evidence indicates that the podocyte slit diaphragm contributes significantly to the size-selective filtration barrier in the kidneys $(36,37)$. Moreover, the disruption of the cytoskeleton leads to foot process effacement in podocytes (38). Herein, to elucidate the mechanisms responsible for the protective effects of NR1 against high glucose-induced podocyte damage, we performed western blot analysis of slit diaphragm proteins and found that NR1 restored the expression levels of these proteins (Fig. 5A). In addition, the results of RT-qPCR (Fig. 5B) also demonstrated that NR1 reduced the expression of desmin and increased the expression of slit diaphragm proteins, which are closely linked to the pathogenesis of foot process effacement under hyperglycemia.

The inhibition of basal autophagy has also been reported to be detrimental to the podocyte architectural structure (22). Maintaining podocyte number plays a pivotal role in the treatment of DN; therefore, in our study, we focused on the anti-apoptotic and autophagy-inducing effects of NR1. Accumulating clinical and experimental data have demonstrated that increased apoptosis and the inhibition of autophagy are important causes of decreased podocyte number $(14,39)$. Therefore, therapies targeting apoptosis and autophagy pathways may stabilize podocyte number and delay the progression of chronic glomerular disease. Our study demonstrated that NR1 preserves the podocyte number by inhibiting apoptosis and inducing autophagy. Firstly, we found that NR1 increased cell viability of HG-treated podocytes in a dose- and time-dependent manner. Furthermore, NR1 protected the HG-treated podocytes from apoptosis, verified by 3 independent methods. Secondly, we found that NR1 was capable of inducing autophagy in HG-treated podocytes, and this effect was inhibited by Beclin siRNA or 3-MA (an autophagy inhibitor). Moreover, the enhancement of autophagy induced by NR1 resulted in elevated expression levels of slit diaphragm proteins and restored the cytoskeleton structure. These data are consistent with the hypothesis that autophagy plays an important role in maintaining podocyte number and function.

To explore the mechanisms underlying the anti-apoptotic effects of NR1, we focused on the caspase pathway. There is evidence to indicate that caspase- 3 is involved in many forms of apoptosis (40). It has been demonstrated that caspase-3 is dispensable for certain forms of apoptosis (41). Therefore, whether caspase pathway is involved in the NR1-induced inhibition of podocyte apoptosis gained our attention. It has been demonstrated that in podocytes, PA caused activation of caspase-3 $(42,43)$. Furthermore, previous studies have indicated that caspase- 3 is activated in podocytes under high glucose conditions, and the activation of caspase- 3 is caused by Bcl-2 insufficiency $(44,45)$. However, to the best of our knowledge, no studies to date have focused on whether Bcl-xL and other proteins in the caspase pathway are involved in the effects of NR1. One of the major findings of our study was that NR1 suppressed apoptosis in a caspasedependent manner. The results of western blot analysis revealed that $\mathrm{Bcl} 2, \mathrm{Bcl}-\mathrm{xL}$ and other proteins in the caspase pathway wre involved in the NR1-induced inhibition of apoptosis. In addition, immunofluorescence staining of cleaved caspase- 3 and caspase-3 activity assay also demonstrated that caspase-3 activity was inhibited in the NR1-treated podocytes. Taken together, by three independent methods, our study demonstrates that the caspase pathway is involved in the NR1-induced inhibition of the apoptosis of podocytes.

Among a wealth of signaling pathways in podocyte protection, the PI3K/Akt signaling pathway is one of the main anti-apoptotic pathways. A growing body of evidence supports the notion that the PI3K/Akt pathway controls the remodeling of the actin cytoskeleton and cell viability $(46,47)$. Studies on podocytes, isolated from diabetic mice, have demonstrated that the insulin-dependent phosphorylation of Akt is impaired, and the dysregulation of Akt phosphorylation is associated with podocyte apoptosis (19). Moreover, in vivo studies have indicated that PI3K p85 interacts with nephrin and CD2AP to form the slit diaphragm protein complex that was associated with a strong activation of Akt (48). Therefore, the PI3K/Akt pathway is involved in both the control of cell number and the maintenance of podocyte biology. In this study, we provide evidence showing that the PI3K/Akt pathway is inactivated after podocyte injury by $\mathrm{HG}$, and is then activated following treatment with NR1. We also proved that NR1 induced podocyte autophagy by the phosphorylation of Akt and mTOR.

In conclusion, our study suggests that NR1 protects podocytes from injury, whose damage and decrement account for proteinuria. NR1 plays an anti-apoptotic and autophagyinducing role in cultured podocytes through the PI3K/Akt/ mTOR signaling. Moreover, NR1 protects slit diaphragm proteins in podocytes under high glucose conditions. Taken together, these findings provide insight into the possible mechanisms through which NR1 treatment may preserve the overall podocyte number and prevent podocyte injury, thereby attenuating the development of glomerulosclerosis and many forms of chronic kidney disease.

\section{Acknowledgements}

The present study was supported by grant no. 81160434 from the National Science Foundation of China, grant no. 2013GXNSFDA019016 from the Guangxi Science Foundation, grant no. 2015JY0183 from the Sichuan Science Foundation, a grant from the Chengdu Science Foundation, grant no. 16ZD0253 from the Sichuan Health and Family Planning Commission Funding, and Sichuan Scientific Research Foundation of the Returned Overseas Chinese Scholars. Funding was also provided from Sichuan Provincial People's Hospital.

\section{References}

1. Mogensen CE: Microalbuminuria as a predictor of clinical diabetic nephropathy. Kidney Int 31: 673-689, 1987.

2. Rudberg S, Persson B and Dahlquist G: Increased glomerular filtration rate as a predictor of diabetic nephropathy - an 8-year prospective study. Kidney Int 41: 822-828, 1992.

3. White KE, Bilous RW; Diabiopsies Study Group: Structural alterations to the podocyte are related to proteinuria in type 2 diabetic patients. Nephrol Dial Transplant 19: 1437-1440, 2004.

4. Kriz W, Gretz N and Lemley KV: Progression of glomerular diseases: is the podocyte the culprit? Kidney Int 54: 687-697, 1998.

5. Dalla Vestra M, Masiero A, Roiter AM, Saller A, Crepaldi G and Fioretto P: Is podocyte injury relevant in diabetic nephropathy? Studies in patients with type 2 diabetes. Diabetes 52: 1031-1035, 2003.

6. Meyer TW, Bennett PH and Nelson RG: Podocyte number predicts long-term urinary albumin excretion in Pima Indians with Type II diabetes and microalbuminuria. Diabetologia 42: 1341-1344, 1999. 
7. Pavenstadt H, Kriz W and Kretzler M: Cell biology of the glomerular podocyte. Physiol Rev 83: 253-307, 2003.

8. Huang G, Lv J, Li T, Huai G, Li X, Xiang S, Wang L, Qin Z, Pang J, Zou B and Wang Y: Notoginsenoside R1 ameliorates podocyte injury in rats with diabetic nephropathy by activating the PI3K/Akt signaling pathway. Int J Mol Med 38: 1179-1189, 2016.

9. Quaggin SE: Transcriptional regulation of podocyte specification and differentiation. Microsc Res Tech 57: 208-211, 2002.

10. White KE, Bilous RW, Marshall SM, El Nahas M, Remuzzi G, Piras G, De Cosmo S and Viberti G: Podocyte number in normotensive type 1 diabetic patients with albuminuria. Diabetes 51: 3083-3089, 2002.

11. Verzola D, Gandolfo MT, Ferrario F, Rastaldi MP, Villaggio B, Gianiorio F, Giannoni M, Rimoldi L, Lauria F, Miji M, et al: Apoptosis in the kidneys of patients with type II diabetic nephropathy. Kidney Int 72: 1262-1272, 2007.

12. Liu WJ, Tang HT, Jia YT, Ma B, Fu JF, Wang Y, Lv KY and $\mathrm{Xia} Z \mathrm{ZF}$ : Notoginsenoside R1 attenuates renal ischemia-reperfusion injury in rats. Shock 34: 314-320, 2010.

13. Gui D, Wei L, Jian G, Guo Y, Yang J and Wang N: Notoginsenoside R1 ameliorates podocyte adhesion under diabetic condition through $\alpha 3 \beta 1$ integrin upregulation in vitro and in vivo. Cell Physiol Biochem 34: 1849-1862, 2014.

14. Hartleben B, Gödel M, Meyer-Schwesinger C, Liu S, Ulrich T, Köbler S, Wiech T, Grahammer F, Arnold SJ, Lindenmeyer MT, et al: Autophagy influences glomerular disease susceptibility and maintains podocyte homeostasis in aging mice. J Clin Invest 120 1084-1096, 2010.

15. Schiffer M, Bitzer M, Roberts IS, Kopp JB, ten Dijke P, Mundel $P$ and Böttinger EP: Apoptosis in podocytes induced by TGF-beta and Smad7. J Clin Invest 108: 807-816, 2001

16. Isermann B, Vinnikov IA, Madhusudhan T, Herzog S, Kashif M, Blautzik J, Corat MA, Zeier M, Blessing E, Oh J, et al: Activated protein $\mathrm{C}$ protects against diabetic nephropathy by inhibiting endothelial and podocyte apoptosis. Nat Med 13: 1349-1358, 2007.

17. Mundel P and Shankland SJ: Podocyte biology and response to injury. J Am Soc Nephrol 13: 3005-3015, 2002

18. Zeng C, Fan Y, Wu J, Shi S, Chen Z, Zhong Y, Zhang C, Zen K and Liu Z: Podocyte autophagic activity plays a protective role in renal injury and delays the progression of podocytopathies. J Pathol 234: 203-213, 2014.

19. Tejada T, Catanuto P, Ijaz A, Santos JV, Xia X, Sanchez P, Sanabria N, Lenz O, Elliot SJ and Fornoni A: Failure to phosphorylate AKT in podocytes from mice with early diabetic nephropathy promotes cell death. Kidney Int 73: 1385-1393, 2008

20. Xiao H, Shi W, Liu S, Wang W, Zhang B, Zhang Y, Xu L, Liang X and Liang Y: 1,25-Dihydroxyvitamin $\mathrm{D}(3)$ prevents puromycin aminonucleoside-induced apoptosis of glomerular podocytes by activating the phosphatidylinositol 3-kinase/Akt-signaling pathway. Am J Nephrol 30: 34-43, 2009.

21. Feng Z, Tang L, Wu L, Cui S, Hong Q, Cai G, Wu D, Fu B, Wei R and Chen $\mathrm{X}: \mathrm{Na}^{+} / \mathrm{H}^{+}$exchanger-1 reduces podocyte injury caused by endoplasmic reticulum stress via autophagy activation. Lab Invest 94: 439-454, 2014.

22. Cinà DP, Onay T, Paltoo A, Li C, Maezawa $\mathrm{Y}$, De Arteaga J, Jurisicova A and Quaggin SE: Inhibition of MTOR disrupts autophagic flux in podocytes. J Am Soc Nephrol 23: 412-420, 2012.

23. Gödel M, Hartleben B, Herbach N, Liu S, Zschiedrich S, Lu S, Debreczeni-Mór A, Lindenmeyer MT, Rastaldi MP, Hartleben G, et al: Role of mTOR in podocyte function and diabetic nephropathy in humans and mice. J Clin Invest 121 2197-2209, 2011.

24. Saleem MA, O'Hare MJ, Reiser J, Coward RJ, Inward CD, Farren T, Xing CY, Ni L, Mathieson PW and Mundel P: A conditionally immortalized human podocyte cell line demonstrating nephrin and podocin expression. J Am Soc Nephrol 13: 630-638, 2002.

25. Li C, Chen J, Lu B, Shi Z, Wang H, Zhang B, Zhao K, Qi W, Bao J and Wang Y: Molecular switch role of Akt in Polygonatum odoratum lectin-induced apoptosis and autophagy in human non-small cell lung cancer A549 cells. PLoS One 9: e101526, 2014.

26. Wang Y, Liu Y, Wang H, Li C, Qi P and Bao J: Agaricus bisporus lectins mediates islet $\beta$-cell proliferation through regulation of cell cycle proteins. Exp Biol Med (Maywood) 237: 287-296, 2012 .
27. Wang Y, Wang H, Liu Y, Li C, Qi P and Bao J: Antihyperglycemic effect of ginsenoside Rh2 by inducing islet $\beta$-cell regeneration in mice. Horm Metab Res 44: 33-40, 2012.

28. Rantanen M, Palmén T, Pätäri A, Ahola H, Lehtonen S, Aström E, Floss T, Vauti F, Wurst W, Ruiz P: Nephrin TRAP mice lack slit diaphragms and show fibrotic glomeruli and cystic tubular lesions. J Am Soc Nephrol 13: 1586-1594, 2002.

29. Kawachi H, Koike H, Kurihara H, Sakai T and Shimizu F: Cloning of rat homologue of podocin: expression in proteinuric states and in developing glomeruli. J Am Soc Nephrol 14: 46-56, 2003.

30. Wiggins JE, Goyal M, Sanden SK, Wharram BL, Shedden KA, Misek DE, Kuick RD and Wiggins RC: Podocyte hypertrophy, 'adaptation,' and 'decompensation' associated with glomerular enlargement and glomerulosclerosis in the aging rat: prevention by calorie restriction. J Am Soc Nephrol 16: 2953-2966, 2005.

31. Schmittgen TD and Livak KJ: Analyzing real-time PCR data by the comparative C(T) method. Nat Protoc 3: 1101-1108, 2008.

32. Reiser J, Kriz W, Kretzler M and Mundel P: The glomerular slit diaphragm is a modified adherens junction. J Am Soc Nephrol 11: $1-8,2000$.

33. Nielsen JS and McNagny KM: The role of podocalyxin in health and disease. J Am Soc Nephrol 20: 1669-1676, 2009.

34. Yaoita E, Kawasaki K, Yamamoto T and Kihara I: Variable expression of desmin in rat glomerular epithelial cells. The Am J Pathol 136: 899-908, 1990.

35. Floege J, Alpers CE, Sage EH, Pritzl P, Gordon K, Johnson RJ and Couser WG: Markers of complement-dependent and complement-independent glomerular visceral epithelial cell injury in vivo. Expression of antiadhesive proteins and cytoskeletal changes. Lab Invest 67: 486-497, 1992.

36. Somlo $S$ and Mundel $P$ : Getting a foothold in nephrotic syndrome. Nat Genet 24: 333-335, 2000

37. Kerjaschki D: Caught flat-footed: podocyte damage and the molecular bases of focal glomerulosclerosis. J Clin Invest 108: 1583-1587, 2001.

38. Saleem MA, Ni L, Witherden I, Tryggvason K, Ruotsalainen V, Mundel P and Mathieson PW: Co-localization of nephrin, podocin, and the actin cytoskeleton: evidence for a role in podocyte foot process formation. Am J Pathol 161: 1459-1466, 2002.

39. Asanuma K, Tanida I, Shirato I, Ueno T, Takahara H, Nishitani T, Kominami E and Tomino Y: MAP-LC3, a promising autophagosomal marker, is processed during the differentiation and recovery of podocytes from PAN nephrosis. FASEB J 17: 1165-1167, 2003.

40. Nunez G, Benedict MA, Hu Y and Inohara N: Caspases: the proteases of the apoptotic pathway. Oncogene 17: 3237-3245, 1998.

41. Mohr S, McCormick TS and Lapetina EG: Macrophages resistant to endogenously generated nitric oxide-mediated apoptosis are hypersensitive to exogenously added nitric oxide donors: dichotomous apoptotic response independent of caspase 3 and reversal by the mitogen-activated protein kinase kinase (MEK) inhibitor PD 098059. Proc Natl Acad Sci USA 95: 5045-5050, 1998

42. Wada T, Pippin JW, Marshall CB, Griffin SV and Shankland SJ: Dexamethasone prevents podocyte apoptosis induced by puromycin aminonucleoside: role of p53 and Bcl-2-related family proteins. J Am Soc Nephrol 16: 2615-2625, 2005.

43. Li X, Zhang X, Li X, Ding F and Ding J: The role of survivin in podocyte injury induced by puromycin aminonucleoside. Int J Mol Sci 15: 6657-6673, 2014.

44. Chen YQ, Wang XX, Yao XM, Zhang DL, Yang XF, Tian SF and Wang NS: MicroRNA-195 promotes apoptosis in mouse podocytes via enhanced caspase activity driven by BCL2 insufficiency. Am J Nephrol 34: 549-559, 2011.

45. Gui D, Guo Y, Wang F, Liu W, Chen J, Chen Y, Huang J and Wang N: Astragaloside IV, a novel antioxidant, prevents glucose-induced podocyte apoptosis in vitro and in vivo. PLoS One 7: e39824, 2012

46. Cantley LC: The phosphoinositide 3-kinase pathway. Science 296: 1655-1657, 2002.

47. Franke TF, Hornik CP, Segev L, Shostak GA and Sugimoto C: PI3K/Akt and apoptosis: size matters. Oncogene 22: 8983-8998, 2003.

48. Huber TB, Hartleben B, Kim J, Schmidts M, Schermer B, Keil A, Egger L, Lecha RL, Borner C, Pavenstädt H, et al: Nephrin and CD2AP associate with phosphoinositide 3-OH kinase and stimulate AKT-dependent signaling. Mol Cell Biol 23: 4917-4928, 2003 